OPEN ACCESS

Edited by:

Sujit Prabhu,

University of Texas MD Anderson

Cancer Center, United States

Reviewed by:

Carsten Friedrich,

University of Rostock, Germany

Yoshua Esquenazi,

University of Texas Health Science

Center at Houston, United States

*Correspondence:

Luis F. Oñate-Ocaña

Ifonate@gmail.com

Specialty section:

This article was submitted to Neuro-Oncology and Neurosurgical

Oncology,

a section of the journa

Frontiers in Neurology

Received: 28 July 2018

Accepted: 11 January 2019

Published: 30 January 2019

Citation:

Cacho-Díaz B,

Lorenzana-Mendoza NA and Oñate-Ocaña LF (2019) Quality of Life in Brain Cancer: Clinical Validation of the Mexican-Spanish Version of the EORTC QLQ-BN20 Questionnaire.

Front. Neurol. 10:40.

doi: 10.3389/fneur.2019.00040

\section{Quality of Life in Brain Cancer: Clinical Validation of the Mexican-Spanish Version of the EORTC QLQ-BN20 Questionnaire}

\author{
Bernardo Cacho-Díaz ${ }^{1}$, Nydia A. Lorenzana-Mendoza ${ }^{1}$ and Luis F. Oñate-Ocaña ${ }^{2 *}$ \\ ${ }^{1}$ Unidad de Neurociencias, Instituto Nacional de Cancerología, Mexico City, Mexico, ${ }^{2}$ Subdirección de Investigación Clínica, \\ Instituto Nacional de Cancerología, Mexico City, Mexico
}

Background: Overall survival (OS) of patients with Brain Cancer (BC) is slowly increasing. The disease itself and its treatments deeply impact patient Health-related quality of life (HRQL). Therefore, valid and reliable instruments are needed. In this study, the Mexican-Spanish version of the QLQ-BN20 instrument is psychometrically and clinically validated.

Methods: Patients with brain cancer (BC) (primary or metastatic) evaluated at a tertiary cancer center, were invited to respond to the questionnaire, as well as the core-module QLQ-C30. Tests to demonstrate the instrument's internal consistency, the association of $H R Q L$ scales with clinical variables and OS were investigated.

Results: One hundred and nineteen patients were included in this cohort: 77 women and 42 men (mean age, 46.2 years). Patients answered both instruments in $<30$ min. Good convergent [all correlation coefficients $(\mathrm{CC})>0.37$ ] and discriminant validity was observed and was associated with significant overlap (CC 0.007-0.68). All four multi-item scales of QLQ-BN20 also demonstrated good reliability (Cronbach $\alpha>0.7)$. Several scales of the QLQ-BN20 were significantly associated with performance status and a modified Recursive Partition Analysis. Of the possible scale correlations, 40 of 161 (24.8\%) scales in both instruments, were significantly (directly or inversely) correlated. Visual disorders, Motor dysfunction, Seizures and Weakness of the legs presented association with OS $(p<0.05)$.

Conclusion: The Mexican-Spanish version of the BN2O instrument is valid and reliable and can be used in clinical trials in patients with BC. Some HRQL scales were associated with OS and could therefore be incorporated in future studies of prognostic models.

Keywords: health-related quality of life, brain neoplasms, surveys and questionnaires, validation study, prognostic factors

\section{INTRODUCTION}

Brain cancer (BC) constitutes a heterogeneous group of diseases, accounting for 1-2\% of all primary cancers in adults (1). These neoplasms (primary or secondary) are characterized by severe and complex symptoms, usually associated with a poor prognosis $(1,2)$. There is no definite cure for most patients. Therefore, a reasonable primary aim of treatment is to extend survival with effective symptom relief $(2,3)$. 
Efforts with emerging new therapeutic strategies are mainly focused on prolonging survival (4). However, BC is associated to symptoms and complications that negatively impact patients' Health-related quality of life (HRQL). BC may directly provoke disabling symptoms including headache, sensory-motor dysfunction, seizures, mood disorders, personality changes, and cognitive dysfunction (5). Consequently, the clinical benefits of treatment should be evaluated not only according to the classical outcome measures (objective response or survival) but also by ensuring HRQL improvement, and must be weighed against treatment side-effects (5-7).

The clinical consequences of disease can be identified by physical examination and evaluated with neurological and neuropsychological tests. Patients' opinions on their own HRQL differ substantially from the opinions of proxies or healthcare personnel. Hence, two instruments have been developed to measure HRQL in the specific case of BC: the FACT-Br, Peds-FACT-BrS, and the European Organization for Research and Treatment of Cancer (EORTC) quality of life questionnaire (QLQ) BN20 (8-11). The MDASI-BT is a symptom inventory designed to measure symptoms and not for HRQL assessment (12).

The QLQ-BN20 measures focus more specifically on function and symptoms, while the FACT-Br assessments cover more psychosocial aspects of the disease. Therefore, the QLQ-BN20 is superior when assessing the treatment outcome and may provide more information in trials that focus on functional endpoints, whereas FACT-Br could be more useful in patients with positive functional capacity but psychosocial concerns, although no instrument is superior to the other (13). Both must be considered as complementary.

The QLQ-BN20 instrument comprises 11 symptom scales that cover the more common complains in patients with $\mathrm{BC}$ and it was designed to be used with the core questionnaire QLQ-C30. This core instrument comprises five functional scales, and nine symptom scales plus a global HRQL scale. Both have been translated and validated into several languages and have been extensively used in the medical literature. However, available information on the subject published in Latin-American countries is scarce. The aim of this study was to validate the Mexican-Spanish version of the QLQ-C30 and QLQ-BN20 questionnaires in patients with BC.

\section{MATERIALS AND METHODS}

\section{Patients}

Patients treated at the Neuro-oncology Unit of the Instituto Nacional de Cancerología (INCan) in Mexico City from February 2005 to October 2014, were invited to participate in this study and respond the questionnaires. Inclusion criteria were: Literate individuals of any gender or age, who had a clinical diagnosis of primary or secondary $\mathrm{BC}$. The diagnosis of $\mathrm{BC}$ was established by computed tomography scan and/or magnetic resonance imaging. The clinical history was obtained, as well as blood cytology and chemistry, tumor markers, and chest-X ray. The Karnofsky and ECOG status performance scales were assessed. The Institutional Review Board and Ethics committees approved the study protocol (registration codes 014/007/CCI and CEI 865/14). Patients signed the informed consent form in which the purpose of the study and a safety protection policy were detailed and accompanied the questionnaires.

\section{Instruments}

The EORTC QLQ-C30 consists of 30 items, which are organized into five functional scales (physical, role, emotional, cognitive, and social functioning); three symptom scales (fatigue, nausea and vomiting, and pain); one global health status scale; and six single items (dyspnea, insomnia, appetite loss, constipation, diarrhea, and financial difficulties). The QLQ-BN20 includes 20 items, which are organized into four multi-item scales (future uncertainty, visual disorders, motor dysfunction, and communication deficit) and seven single items (headache, seizures, drowsiness, hair loss, itchy skin, weakness of the legs, and bladder control) (11). The validated Mexican-Spanish version of the QLQ-C30 was used (14). The adapted MexicanSpanish version of the QLQ-BN20 was pilot-tested in 10 patients with $\mathrm{BC}$ to identify the adequacy of the translation. All patients responded the questionnaire without assistance, stated that the questions were clear and easy to understand and complete. Both questionnaires were used with permission of the EORTC Quality of Life Group, and they supervised the entire process.

\section{Statistical Analysis}

Scale scores were calculated by linear transformation of raw scores into a $0-100$ score, with 100 representing best global health, best functional status, or worst symptoms, as described by EORTC (15), and the summary score was also calculated (16). Correlation assessment was obtained with the Spearman Correlation coefficient (CC). Convergent validity was determined calculating the CC between each item and items belonging to their own scale, and the success criteria was $\mathrm{CC}>0.3$. Divergent validity was evaluated calculating the CC between each item and items belonging to other scales, and the success criteria was $\mathrm{CC}<0.3$. Cronbach's $\alpha$ was used to measure multi-item correlation, and the success criteria was $\geq 0.7$. In addition, clinical validity was evaluated by the extent to which scores were able to discriminate among groups of patients who differed in terms of their clinical status. Patients were classified according to treatment intent, the number of metastases (single, multiple or carcinomatosis) and according to Recursive Partitioning Analysis (RPA) (17). Due to the heterogeneity of primary BC and the limited availability of specific prognostic scores for each of the different types of $\mathrm{BC}$, for the purpose of the study, patients with primary BC were classified using the same RPA approach, as if it were metastatic, considering the absence of neoplasm in sites other than the CNS, age ( $>65$ years) and a Karnofsky score $>70$ $(18,19)$.

The Kruskal-Wallis test was used to analyze differences between groups. Scale scores were categorized by terciles. The correlation between the different QLQ-C30 and QLQBR20 scales was explored to identify differences and clinical overlapping. Overall survival (OS) was considered as the period of time from diagnosis of $\mathrm{BC}$ metastases or primary $\mathrm{BC}$ to death. The association of HRQL and OS was evaluated using 
the Kaplan-Meier method, and differences were tested with the Log rank method. Hazard ratios (HR) were calculated using the Cox model. Sample-size calculation was based on the proposal by Tabachnik and Fidell (20); a minimal ratio of 5 patients per item was required $(20 \times 5)$, i.e., a sample size of 100 patients. Any probability value of 0.05 or less was considered significant, and two-tailed statistics were applied in all cases. The SPSS for Mac version 23 software was used for computations (IBM, Inc., Armonk, NY, USA).

\section{RESULTS}

\section{Patients}

One hundred twenty-seven (127) patients were invited to participate, but eight did not consent. Therefore, 119 patients with $\mathrm{BC}$ were included in the study. There were 77 women $(64.7 \%)$ and 42 men (35.3\%), with a mean age of 46.18 years (SD,
15.8; range 17-80). Brain metastases were found in 76 patients (63.9\%); among these, the most common primary cancer sites were: 25 breast (32.9\%), 18 lung (23.7\%), four ovary (5.3\%), four non-Hodgkin lymphoma, three Hodgkin's lymphoma (3.9\%), three cervix-uteri, three renal, three testicle, two melanoma (2.6\%), two endometrial, one acute myeloid leukemia (1.3\%), one gastric, one prostate, one metastases to spinal cord from a treated medulloblastoma, one meningeal metastases from a treated medulloblastoma, one rectum, one nasopharynx, one adenoid cystic and one basocellular skin cancer. Primary BC was found in 43 patients $(36.1 \%)$ and the most frequent diagnoses were: 12 meningioma (27.9\%), six astrocytoma (13.9\%), five CNS primary germinal neoplasms, four primary CNS lymphoma, four medulloblastoma (9.3\%), four high-grade glioma, three pituitary macroadenoma, two oligoastrocytoma, one craniopharyngioma, one ependymoma, one gliosarcoma, one hemangiopericytoma, one oligodendroglioma, one meningeal sarcoma and one

TABLE 1 | Descriptive statistics of EORTC QLQ-C30 and QLQ-BN20 functional and symptom scales in patients with (primary or secondary) brain cancer ( $n=119$ ).

\begin{tabular}{|c|c|c|c|c|}
\hline & Mean (SD) & Median & Floor (\%) & Ceiling (\%) \\
\hline \multicolumn{5}{|l|}{ QLQ-C30 } \\
\hline Global health/QoL & $66.96(28.1)$ & 66.67 & $0(1.6)$ & $100(18.3)$ \\
\hline \multicolumn{5}{|l|}{ Functional scales } \\
\hline Physical & $72.67(27)$ & 80 & $0(1.6)$ & $100(16.7)$ \\
\hline Role & 73.61 (32.9) & 83.33 & $0(8.7)$ & $100(43.7)$ \\
\hline Emotional & 72.62 (22.5) & 75 & $0(0.8)$ & $100(15.9)$ \\
\hline Cognitive & 75.56 (19.8) & 83.33 & $16.67(2.4)$ & $100(94.4)$ \\
\hline Social & 73.52 (29.7) & 83.33 & $0(5.6)$ & $100(37.3)$ \\
\hline \multicolumn{5}{|l|}{ Symptom scales } \\
\hline Fatigue & 32.24 (23.9) & 33.33 & $0(11.1)$ & $100(1.6)$ \\
\hline Nausea and vomiting & 12.93 (20.9) & 0 & 0 (60.3) & $100(0.8)$ \\
\hline Pain & $23.11(24.8)$ & 16.67 & $0(34.9)$ & $100(1.6)$ \\
\hline Dyspnea & $26.89(30.5)$ & 0 & $0(65.9)$ & $100(2.4)$ \\
\hline Insomnia & $26.89(30.5)$ & 33.33 & $0(43.7)$ & $100(6.3)$ \\
\hline Appetite loss & $20.73(29.7)$ & 0 & 0 (56.3) & $100(5.6)$ \\
\hline Constipation & $27.45(30.6)$ & 33.33 & $0(43.7)$ & $100(5.6)$ \\
\hline Diarrhea & $11.76(24.8)$ & 0 & 0 (72.2) & $100(4)$ \\
\hline Financial difficulties & 47.06 (39.6) & 33.33 & $0(28.6)$ & $100(26.2)$ \\
\hline Summary score & 76.9 (17.04) & 81.15 & $26.11(0.8)$ & $100(1.7)$ \\
\hline \multicolumn{5}{|l|}{ QLQ-BN20 } \\
\hline \multicolumn{5}{|l|}{ Symptom scales } \\
\hline Future uncertainty & $28.36(25.5)$ & 25 & $0(17.5)$ & $100(1.6)$ \\
\hline Visual disorders & $24.09(26.1)$ & 22.22 & 0 (33.3) & $100(2.4)$ \\
\hline Motor dysfunction & $26.8(26)$ & 22.22 & $0(27)$ & $100(0.8)$ \\
\hline Communication deficit & 21.57 (25.2) & 11.11 & 0 (38.9) & $100(0.8)$ \\
\hline Headache & 25.58 (29.3) & 33.33 & $0(43.7)$ & $100(5.6)$ \\
\hline Seizures & $5.88(19.7)$ & 0 & $0(84.9)$ & $100(2.4)$ \\
\hline Drowsiness & 33.05 (31.4) & 33.33 & $0(32.5)$ & $100(9.5)$ \\
\hline Hair loss & $20.45(30.41)$ & 0 & $0(57.9)$ & $100(6.3)$ \\
\hline Itchy skin & 18.77 (27.3) & 0 & $0(57.9)$ & $100(3.2)$ \\
\hline Weakness of legs & $28.01(30.1)$ & 33.33 & $0(40.5)$ & $100(6.3)$ \\
\hline Bladder control & $16.25(27.7)$ & 0 & $0(64.3)$ & $100(4.8)$ \\
\hline
\end{tabular}

n, number of patients; SD, standard deviation; \%, is the percentage of patients with floor or ceiling value. 
TABLE 2 | Convergent and discriminant validity of scales of the EORTC QLQ-C30 and QLQ-BN20 instruments in patients with (primary or secondary) brain cancer $(n=119)$.

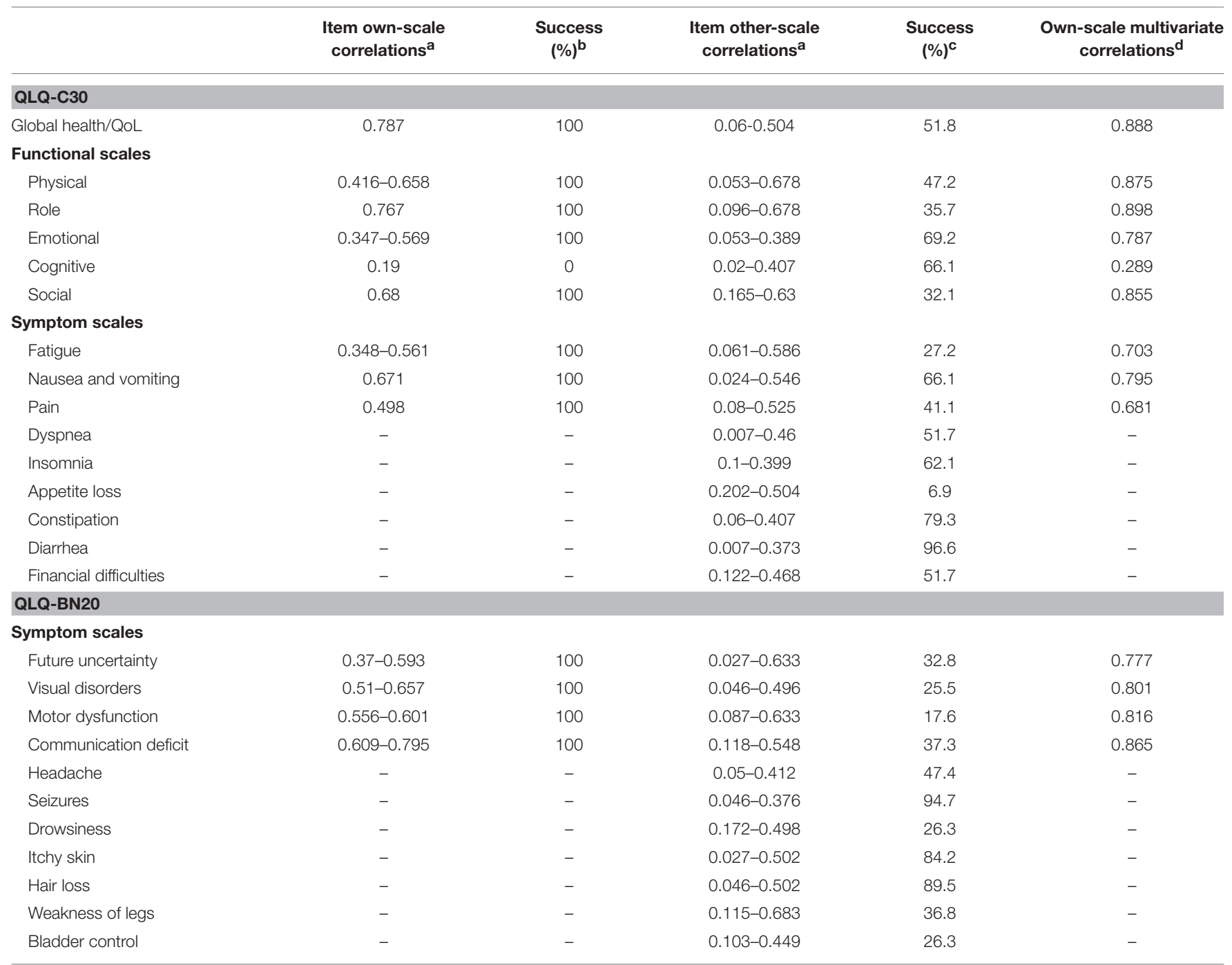

n, number of patients; ${ }^{a}$ Spearman correlation coefficients; ${ }^{b}$ success criteria for item own-scale correlations $(>0.3) ;{ }^{c}$ success criteria for item other-scale correlations ( $\left.<0.3\right) ;{ }^{d}$ Cronbach $\alpha$ values. All correlation coefficient values are absolute values.

pinealoblastoma. Among patients with primary $\mathrm{BC}$, one developed breast cancer as a second primary, with brain metastases and meningioma; one patient had a retroauricular mucoepidermoid carcinoma with a metachronous astrocytoma and meningioma; in two patients with primary medulloblastoma, one had meningeal metastases and the other developed spinal cord involvement.

Six patients had a 100\% KPS, $3990 \%, 4180 \%$, nine $70 \%$ and $2760 \%$ or below KPS. All patients answered both questionnaires in $<30 \mathrm{~min}$, and there were 6 missing values $(0.1 \%)$ in the 50 items of both instruments, including 5,950 possible responses.

\section{Reliability and Internal Validity}

Descriptive statistics of the HRQL data are presented in Table $\mathbf{1 .}$ Most scales of both instruments have a zero floor and a 100 ceiling values, whereby mean scores mainly represent highfunctional and low-symptom values. The summary of multi-trait scaling analyses is depicted in Table 2; good convergent and discriminant validity is observed for most scales. All multi-item scales presented good own-scale correlations. Divergent validity revealed low correlation for other-scale items but also frequent overlapping. The Cognitive and Pain scales of the QLQ-C30 did not show a Cronbach's $\alpha$ coefficient $>0.70$, but all four multi-item scales of the QLQ-BN20 did.

\section{Clinical Validity}

Many scales of both instruments were significantly associated with clinically relevant factors. Table 3 describes the association of three categories of ECOG with scale scores of both instruments; nine of Sixteen (including summary score), and four of Eleven scales of the QLQ-C30 and QLQ-BN20, respectively, 
TABLE 3 | Mean scale scores of QLQ-C30 and QLQ-BN20 depending on the Eastern Cooperative Oncology Group (ECOG) performance status grading in patients with (primary or secondary) brain cancer $(n=119)$.

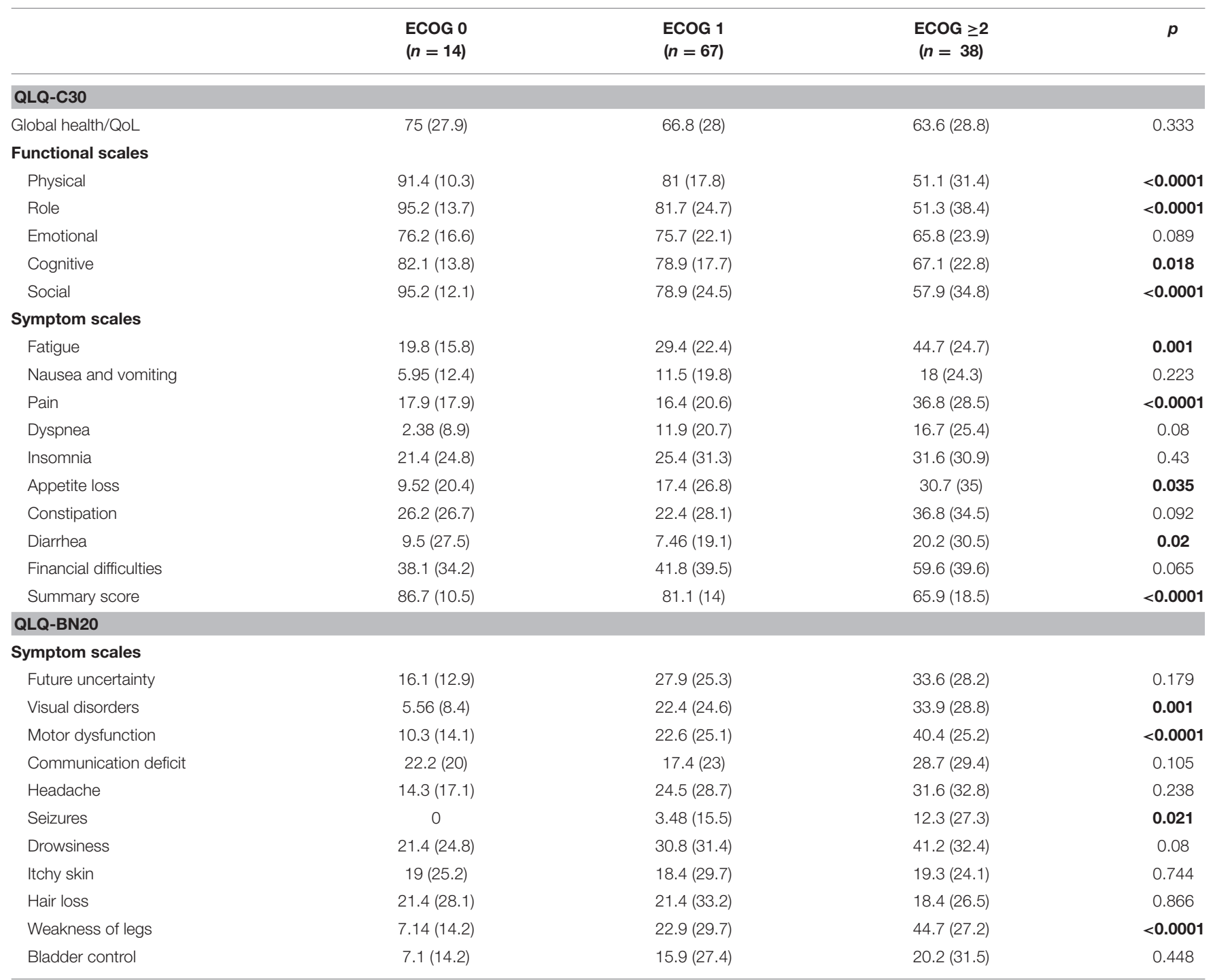

n, number of patients; numbers represent means (in parentheses are standard deviation values); p, probability values obtained by Kruskal-Wallis test. Bold values correspond to statistical significant values.

showed significant associations. Table 4 shows the association of RPA categories (for metastatic and primary BC) with the mean scale scores of both instruments; three of 16 (including summary score), and three of 11 scales of the QLQ-C30 and QLQ-BN20, respectively, did not yield significant associations.

\section{Correlations Between Instruments}

Forty-two of $176(23.8 \%)$ possible $(16 \times 11)$ bivariate correlations between QLQ-C30 and QLQ-BN20 scales were significant $(p<0.05)$. As expected, correlation between functional and symptom scales were usually negative. The correlation matrix is shown in the Table 5.

\section{Survival}

Median follow-up of the cohort was 4.49 years (SD 3.38) (range 0.21-13.8). During this period, 79 patients (66.4\%) died from progressive or recurrent disease. Median OS was 3.98 years (95\% CI 2.99-4.97). The bivariate association of HRQL and OS were explored; the physical, role, social, fatigue, nausea/vomiting, pain, dyspnea, appetite loss, financial difficulties scales, and the summary score were associated with OS. Of the QLQ-BN20 scales, Visual disorders (HR 1.01 [95\%CI 1.002-1.018]), Motor dysfunction (HR 1.011 [95\% CI 1.003-1.019]), Seizures (HR 1.013 [95\% CI 1.004-1.023]), and Weakness of the legs (HR 1.013 [95\% CI 1.007-1.02]) were associated with OS. The Kaplan-Meier OS curves depending on Visual disorders, Motor dysfunction, Seizures and Weakness of the legs scales are depicted in Figure 1.

\section{DISCUSSION}

In this study, the Mexican-Spanish version of the QLQ-BN20 instrument along with its core instrument QLQ-C30 has been 
TABLE 4 | Mean scale scores of QLQ-C30 and QLQ-BN20 depending on the RPA class in patients with (primary or secondary) brain cancer ( $n=119)$.

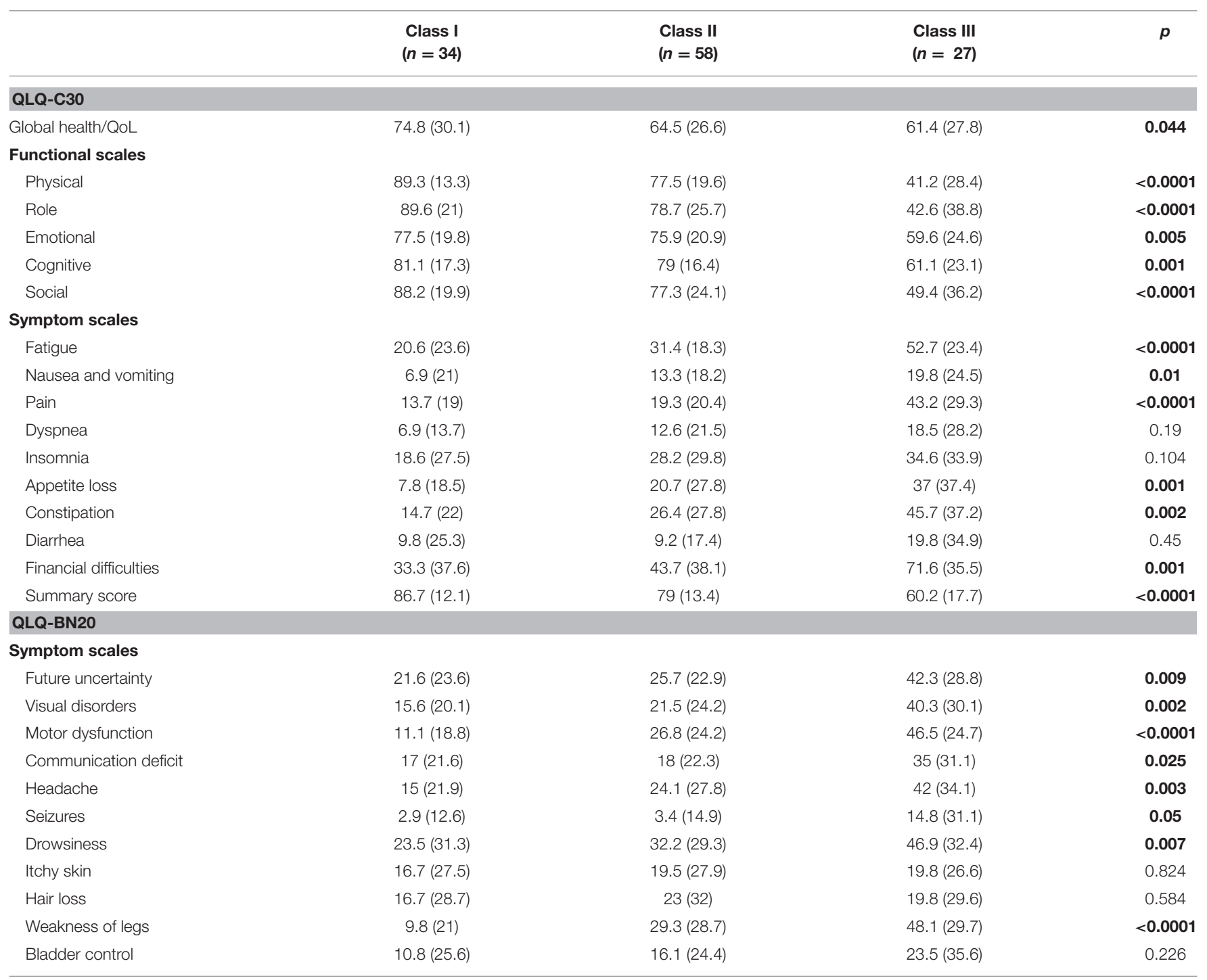

n, number of patients; numbers represent means (in parentheses are standard deviation values); $p$, probability values obtained by Kruskal-Wallis test. Bold values correspond to statistical significant values.

psychometrically and clinically validated as we found them reliable and valid.

The main traditional outcome measures in oncology research are the frequency of objective responses after therapy, progression-free survival, or OS. However, many brain neoplasms are incurable, and maintenance or improvement of patients' HRQL are, at least, as important as increases in the progression-free survival or OS. On the other hand, a patient-centered approach complementing the decision-making process in Neuro-Oncology is feasible and desirable. Most patients with $\mathrm{BC}$ can participate actively in the decisions on their management options if relevant information is presented in a clear and reasonable manner. When informed, most patients are able to identify concepts of HRQL, the capability to maintain functional independence and the influence of treatment on survival as the most relevant factors in determining their decision (21). As physicians, we must be prepared to facilitate this process.

In a 20-year period, only five Randomized clinical trials (RCT) included HRQL evaluations as primary or secondary outcome measurements. However, the quality of reporting HRQL data has not considerably improved (22). In these contexts, the availability of valid instruments to accurately measure HRQL is mandatory. In general terms, the psychometric characteristics of our study were similar to the original report and other validation reports $(23,24)$.

The original QLQ-BN20 instrument was developed in multilingual and multicultural settings in Europe (including European-Spanish) and has proven to be valid and reliable $(11,25)$. Our study is the first validation protocol of the instrument in the Mexican-Spanish language, performed in a Cancer Center in Latin America. Similar psychometric findings 
TABLE 5 | Correlation matrix of QLQ-C30 and QLQ-BN20 mean scale scores in patients with (primary or secondary) brain cancer $(n=119)$.

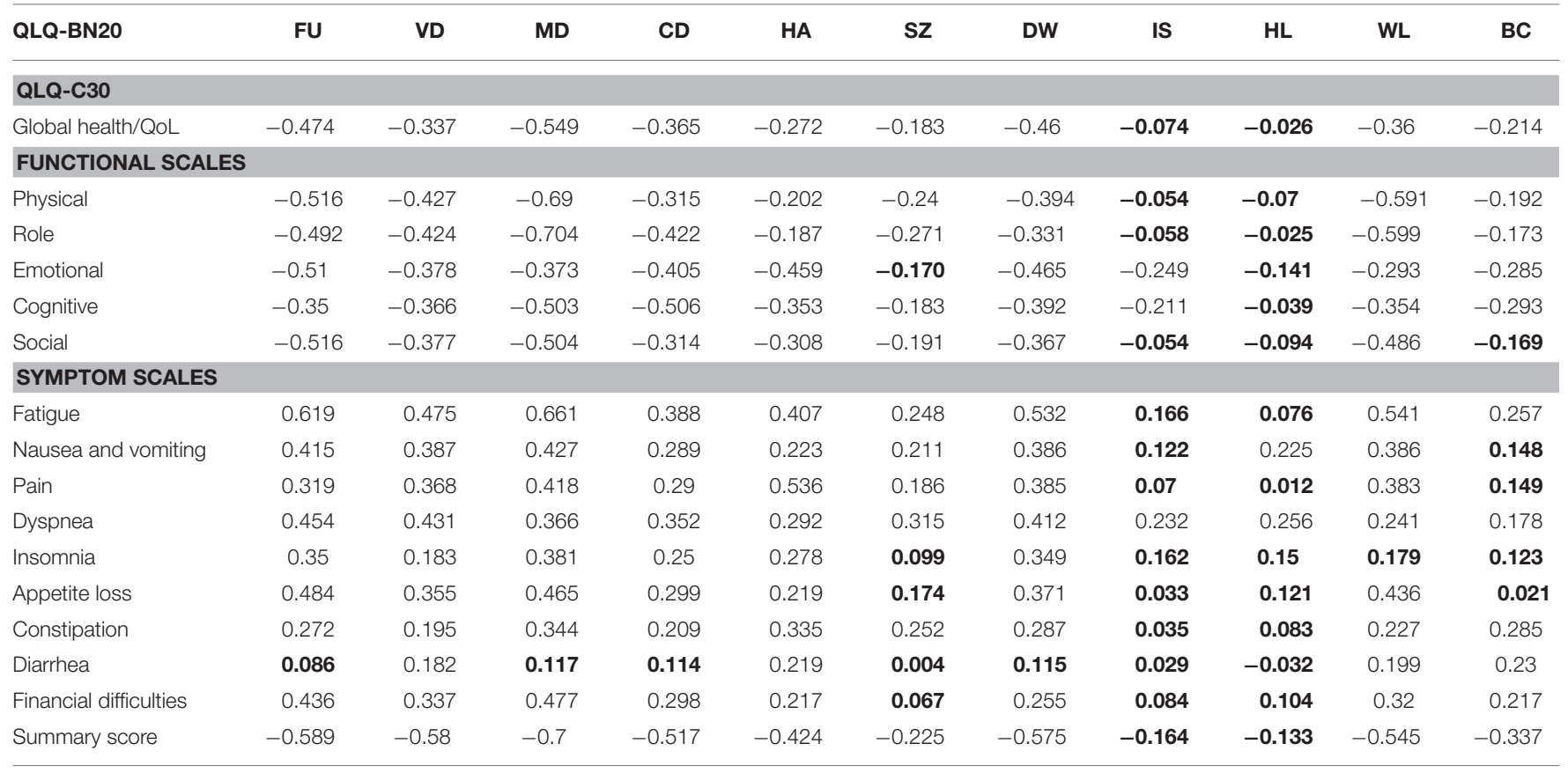

n, number of patients; numbers represent means (in parentheses are standard deviation values); $F U$, future uncertainty; $V D$, visual disorders; $M D$, motor dysfunction; $C D$, communication deficit; HA, headache; SZ, seizures; DW, drowsiness; IS, itchy skin; HL, hair loss; WL, weakness of legs; BC, bladder control.

Non-significant correlations are shown in bold numbers $(p>0.05)$.

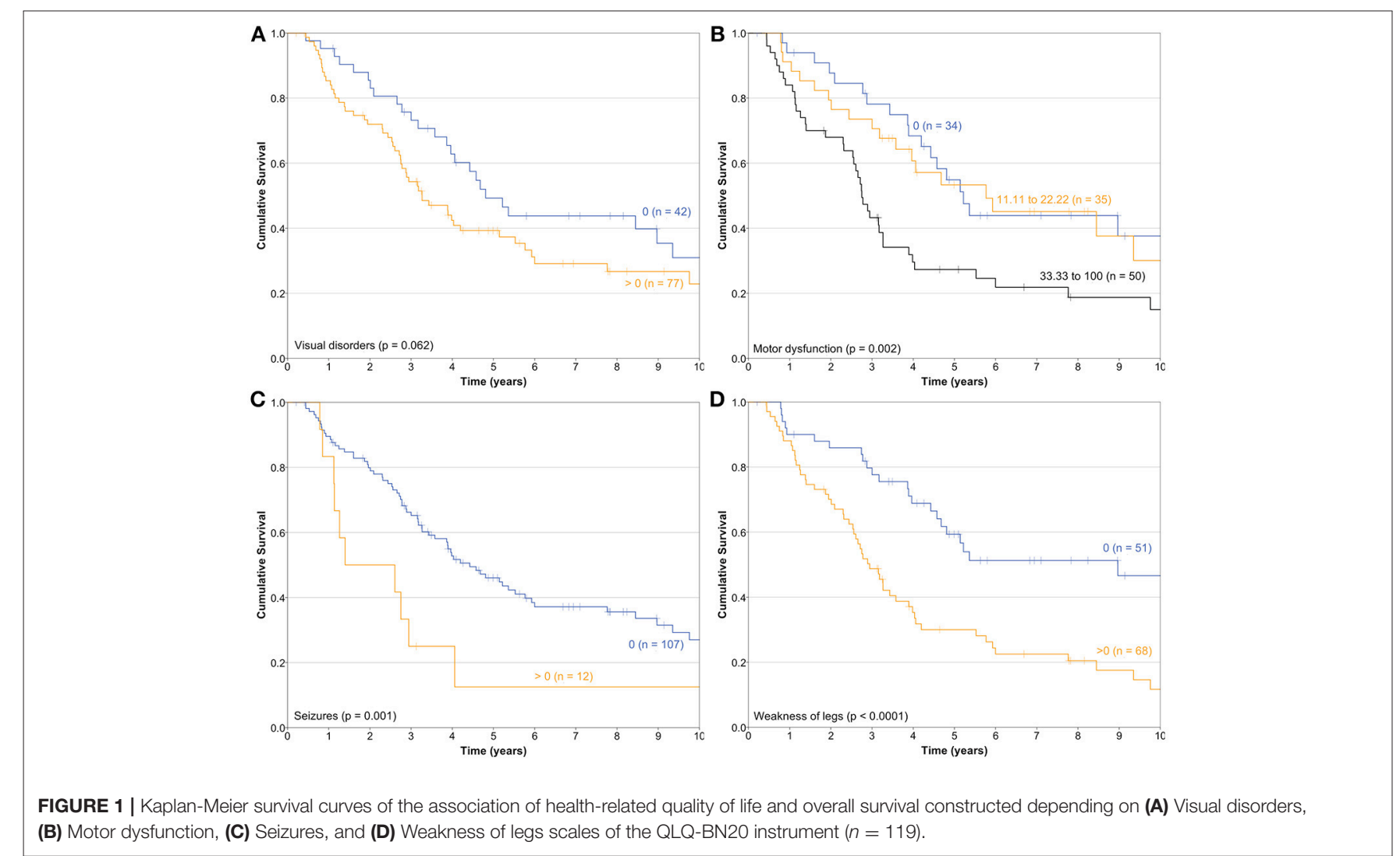


are described in other validation studies but the association of several HRQL scales and relevant clinical variables has not been previously reported $(11,23-26)$. In known-group comparisons, the association of the QLQ-BN20 instrument with the ECOG performance status scale, RPA and OS are described.

The main pitfalls of our study are that responsiveness to cancer treatment was not investigated as long as we have performed one HRQL evaluation for each patient, and our relatively small sample size $(n=119)$, when compared with the original report from the EORTC $(n=891)(11)$.

Floor and ceiling values are $0-100$ in all scales except in the Cognitive scale of the QLQ-C30 (Table 1). In general terms, most patients report high functional and low symptom scales (Table 1), reflecting a population with a recent diagnosis and low disease burden. Convergent and divergent validity is adequate for both instruments, as shown in Table 2 , and as similarly reported in the other four validation studies $(11,23-26)$. The reliability of the Cognitive scale of the QLQ-C30 is below 0.7 and this finding is similar to the Korean validation study (24), while the other three validation studies did not mention the values of the QLQ-C30 scales $(11,23,25,26)$. Low Cronbach $\alpha$ values in terms of Cognitive scale's reliability are frequent in the literature in patients with diverse types of cancer. Examples of this finding include the original EORTC report of the QLQ-C30 instrument (27), and the original Mexican-Spanish validation study of the QLQ-C30 instrument (14). All multi-item scales of the QLQBN20 presented fair Cronbach $\alpha$ values as in the other three validation reports $(11,23-26)$. Certain QLQ-BN20 mean scale scores revealed important associations with ECOG, RPA and OS (Tables 3, 4 and Figure 1). No comparison is possible because to our knowledge, these findings have not been previously reported.

Although, there is currently no available cure for advanced $\mathrm{BC}$, survival rates have been increasing over the last few years, so this tool is useful in assessing the development of an effective treatment that improves HRQL (5). The QLQ-BN20 instrument has been cited in 42 publications in PubMed since 2009 and has been used in clinical trials to measure HRQL in BC patients undergoing chemotherapy $(\mathrm{Ch})$ and/or radiotherapy (RT). A recent study compared two treatment outcomes in glioblastoma patients: one received RT alone, while the other group of patients was treated with RT plus adjuvant temozolomide; QLQ-C30 and QLQ-BN20 were used to assess the patients at follow-up and results showed similar HRQL in the two groups with minimal differences in the nausea/vomiting and constipation scales, which were worse in the Ch / RT group than in the RT only group. Nevertheless, the use of adjuvant temozolomide therapy further prolonged patient survival compared to the RT only group (28).

In clinical trials, statistically significant changes in HRQL can be observed by increasing the sample size or in the scenario of multiple comparisons (such as comparison of multiple HRQL scale scores). However, these changes may not be clinically relevant. The meaning of the minimal clinical important difference is pertinent in the design of clinical trials, when proposing an adequate sample size and in the correct interpretation of results. The minimal clinical important difference can be defined as the smallest difference in the mean score which is clinically important (as in between groups or paired comparison designs). In a recent study, a decrease of 6.1 units or 13.8 units was required to represent clinically relevant deterioration of the Seizures or Weakness of legs variables, respectively (29).

In another study of BC patients, 5.2 units change represented the minimal clinically important deterioration in the motor dysfunction scale. Similarly, 9.1 units change represented clinically important improvement in the communication deficit (30).

In general terms, the authors consider that any 10-unit change or difference in the mean score represents a clinically important difference.

Most patients do not report problems with the cognitive functioning scale. This problem may result from their sociocultural background. At the INCan hospital, we mainly treat patients with a low income, illiteracy, a low education level and poor working possibilities.

Distinguishing patients with glioma from those with meningioma was not tested in this study because of the great variability of histopathology diagnoses in the cohort. This is a validation study, so we did not test the impact of different treatments on HRQL. This question and others could be investigated in future research studies, including the usefulness of these instruments in revealing subtle differences associated to novel treatments in randomized clinical trials.

\section{CONCLUSION}

In conclusion, the Mexican-Spanish version of the QLQ-BN20 instrument is a valid and reliable test that can be used in clinical studies that include patients with primary or metastatic BC. Some HRQL items were associated with the OS and could be used as prognostic factors or might contribute to assemble prognostic models as aids in treatment trade-offs.

\section{AUTHOR CONTRIBUTIONS}

BC-D and LO-O conception and design of the study and analysis and interpretation of data and draft and revise the article. NL-M recruiting patients, acquisition of data, cleaning of the database. All authors have approved the final version to be submitted.

\section{FUNDING}

The INCan financially supported this study in its entirety.

\section{ACKNOWLEDGMENTS}

Many thanks to Professor Deborah Dion Aleman-Hoey for her kind help and English language editorial review. 


\section{REFERENCES}

1. Siegel RL, Miller KD, Jemal A. Cancer statistics, 2018. CA Cancer J Clin. (2018) 68:7-30. doi: 10.3322/caac.21442

2. de Robles P, Fiest KM, Frolkis AD, Pringsheim T, Atta C, St GermaineSmith C, et al. The worldwide incidence and prevalence of primary brain tumors: a systematic review and meta-analysis. Neuro Oncol. (2015) 17:77683. doi: 10.1093/neuonc/nou283

3. Hayat MA. Brain Tumors: Tumors of the Central Nervous System. Vol. 3. New York, NY: Springer (2011).

4. Schiff D, Lee EQ, Nayak L, Norden AD, Reardon DA, Wen PY. Medical management of brain tumors and the sequelae of treatment. Neuro Oncol. (2014) 17:488-504. doi: 10.1093/neuonc/nou304.

5. Dirven L, Reijneveld JC, Taphoorn MJ. Health-related quality of life or quantity of life: a difficult trade-off in primary brain tumors? Semin Oncol. (2015) 41:541-52. doi: 10.1053/j.seminoncol.2014.06.002

6. Lin NU, Wefel JS, Lee EQ, Schiff D, van den Bent MJ, Soffietti R, et al. Challenges relating to solid tumor brain metastases in clinical trials, part 2: neurocognitive, neurological, and quality-of-life outcomes. A report from the RANO group. Lancet Oncol. (2013) 14:e407-16. doi: 10.1016/S1470-2045(13)70308-5

7. Bottomley A, Pe M, Sloan J, Basch E, Bonnetain F, Calvert M, et al. Setting International Standards in Analyzing Patient-Reported Outcomes and Quality of Life Endpoints Data (SISAQOL) consortium. Analyzing data from patientreported outcome and quality of life endpoints for cancer clinical trials: a start in setting international standards. Lancet Oncol. (2016) 17:e510-4. doi: 10.1016/S1470-2045(16)30510-1

8. Weitzner MA, Meyers CA, Gelke CK, Byrne KS, Cella DF, Levin VA. The functional assessment of cancer therapy (FACT) scale. Development of a brain subscale and revalidation of the general version (FACT-G) in patients with primary brain tumors. Cancer (1995) 75:1151-61.

9. Lai JS, Cella D, Tomita T, Bode RK, Newmark M, Goldman S. Developing a health-related quality of life instrument for childhood brain tumor survivors. Child Nervous Syst. (2007) 23:47-57. doi: 10.1007/s00381-006-0176-6

10. Osoba D, Aaronson NK, Muller M, Sneeuw K, Hsu MA, Yung WK, et al. The development and psychometric validation of a brain cancer qualityof-life questionnaire for use in combination with general cancer-specific questionnaires. Qual Life Res. (1996) 5:139-50.

11. Taphoorn MJ, Claassens L, Aaronson NK, Coens C, Mauer M, Osoba $\mathrm{D}$, et al. An international validation study of the EORTC brain cancer module (EORTC QLQ-BN20) for assessing health-related quality of life and symptoms in brain cancer patients. Eur J Cancer (2010) 46:1033-40. doi: 10.1016/j.ejca.2010.01.012

12. Armstrong TS, Mendoza T, Gring I, Coco C, Cohen MZ, Eriksen L, et al. Validation of the MD Anderson symptom inventory brain tumor module (MDASI-BT). J Neurooncol. (2006) 80:27-35. doi: 10.1007/s11060-006-9135-Z

13. Chow R, Lao N, Popovic M, Chow E, Cella D, Beaumont J, et al. Comparison of the EORTC QLQ-BN20 and the FACT-Br quality of life questionnaires for patients with primary brain cancers: a literature review. Support Care Cancer (2014) 22:2593-8. doi: 10.1007/s00520-014-2352-7

14. Oñate-Ocana LF, Alcántara-Pilar A, Vilar-Compte D, García-Hubard G, Rojas-Castillo E, Alvarado-Aguilar S, et al. Validation of the Mexican Spanish version of the EORTC C30 and STO22 questionnaires for the evaluation of health-related quality of life in patients with gastric cancer. Ann Surg Oncol. (2009) 16:88-95. doi: 10.1245/s10434-008-0175-9

15. Fayers PM, Aaronson NK, Bjordal K, Curran D, Groenvold M. EORTC QLQ-C30 Scoring Manual. 3rd Ed. Brussels: EORTC (2001).

16. Giesinger JM, Kieffer JM, Fayers PM, Groenvold M, Petersen MA, Scott NW, et al. EORTC Quality of Life Group. Replication and validation of higher order models demonstrated that a summary score for the EORTC QLQ-C30 is robust. J Clin Epidemiol. (2016) 69:79-88. doi: 10.1016/j.jclinepi.2015.08.007

17. Sperduto PW, Berkey B, Gaspar LE, Mehta M, Curran W. A new prognostic index and comparison to three other indices for patients with brain metastases: an analysis of 1,960 patients in the RTOG database. Int J Radiat Oncol Biol Phys. (2008) 70:510-4. doi: 10.1016/j.ijrobp.2007.06.074

18. Bell EH, Pugh SL, McElroy JP, Gilbert MR, Mehta M, Klimowicz AC, et al. Molecular-based recursive partitioning analysis model for glioblastoma in the temozolomide era: a correlative analysis based on NRG oncology RTOG 0525. JAMA Oncol. (2017) 3:784-92. doi: 10.1001/jamaoncol.2016.6020

19. Abrey LE, Ben-Porat L, Panageas KS, Yahalom J, Berkey B, Curran W, et al. Primary central nervous system lymphoma: the Memorial SloanKettering Cancer Center prognostic model. J Clin Oncol. (2006) 24:5711-5. doi: 10.1200/JCO.2006.08.2941

20. Tabachnik BG, Fidell LS. Using Multivariate Statistics. Boston, MA: Pearson (2007).

21. Zeng KL, Raman S, Sahgal A, Soliman H, Tsao M, Wendszicki C, et al. Patient preference for stereotactic radiosurgery plus or minus whole brain radiotherapy for the treatment of brain metastases. Ann Palliat Med. (2017) 6:S155-60. doi: 10.21037/apm.2017.06.11

22. Dirven L, Taphoorn MJ, Reijneveld JC, Blazeby J, Jacobs M, Pusic A, et al. The level of patient-reported outcome reporting in randomised controlled trials of brain tumour patients: a systematic review. Eur J Cancer (2014) 50:2432-48. doi: 10.1016/j.ejca.2014.06.016

23. Khoshnevisan A, Yekaninejad MS, Ardakani SK, Pakpour AH, Mardani A, Aaronson NK. Translation and validation of the EORTC brain cancer module (EORTC QLQ-BN20) for use in Iran. Health Qual Life Outcomes (2012) 10:54. doi: 10.1186/1477-7525-10-54

24. Shin YS, Kim JH. Validation of the Korean version of the European Organization for Research and Treatment of Cancer brain cancer module (EORTC QLQ-BN20) in patients with brain tumors. Health Qual Life Outcomes (2013) 11:145. doi: 10.1186/1477-7525-11-145

25. Osoba D, Aaronson NK, Muller M, Sneeuw K, Hsy MA, Yung WK, et al. The development and psychometric validation of a brain cancer qualityof-life questionnaire for use in combination with general cancer-specific questionnaires. Qual Life Res. (1996) 5:139-50.

26. Zhang K, Tian J, He Z, Sun W, Pekbay B, Lin Y, et al. Validation of the Chinese version of EORTC QLQ-BN20 for patients with brain cancer. Eur J Cancer Care (2018) 27:e12832. doi: 10.1111/ecc.12832

27. Aaronson NK, Ahmedzai S, Bergman B, Bullinger M, Cull A, Duez NJ, et al. The European Organization for Research and Treatment of Cancer QLQ-C30: a quality-of-life instrument for use in international clinical trials in oncology. J Natl Cancer Inst. (1993) 85:365-76.

28. Perry JR, Laperriere N, O' Callaghan CJ, Brandes AA, Menten J, Phillips C, et al. Short-course radiation plus temozlomide in elderly patients with glioblastoma. $N$ Engl J Med. (2017) 376:1027-37. doi: 10.1056/NEJMoa1611977

29. Wong E, Zhang L, Kerba M, Amalot PF, Danielson B, Tsao M, et al. Minimal clinically important differences in the EORTC QLQ-BN20 in patients with brain metastases. Support Care Cancer (2015) 23:2731-7. doi: 10.1007/s00520-015-2637-5

30. Maringwa J, Quinten C, King M, Ringash J, Osoba D, Coens C, et al. Minimal clinically meaningful differences for the EORTC QLQ-C30 and EORTC QLQ-BN20 scales in brain cancer patients. Ann Oncol. (2011) 22:2107-12. doi: 10.1093/annonc/mdq726

Conflict of Interest Statement: The authors declare that the research was conducted in the absence of any commercial or financial relationships that could be construed as a potential conflict of interest.

Copyright (C) 2019 Cacho-Díaz, Lorenzana-Mendoza and Oñate-Ocaña. This is an open-access article distributed under the terms of the Creative Commons Attribution License (CC BY). The use, distribution or reproduction in other forums is permitted, provided the original author(s) and the copyright owner(s) are credited and that the original publication in this journal is cited, in accordance with accepted academic practice. No use, distribution or reproduction is permitted which does not comply with these terms. 